\title{
3D Modeling of Missing Pellet Surface Defects in BWR Fuel
}

B. W. Spencer*, R. L. Williamson, D. S. Stafford ${ }^{\S}$, S. R. Novascone, J. D. Hales, G. Pastore 


\title{
3D Modeling of Missing Pellet Surface Defects in BWR Fuel
}

\author{
B. W. Spencer ${ }^{\mathrm{a}, *}$, R. L. Williamson ${ }^{\mathrm{a}}$, D. S. Stafford ${ }^{\mathrm{a}}$, S. R. Novascone ${ }^{\mathrm{a}}$, J. D. Hales ${ }^{\mathrm{a}}$, G. Pastore ${ }^{\mathrm{a}}$ \\ ${ }^{a}$ Fuel Modeling and Simulation, Idaho National Laboratory, P.O. Box 1625, Idaho Falls, ID 83415-3840
}

\begin{abstract}
One of the important roles of cladding in light water reactor fuel rods is to prevent the release of fission products. To that end, it is essential that the cladding maintain its integrity under a variety of thermal and mechanical loading conditions. Local geometric irregularities in fuel pellets caused by manufacturing defects known as missing pellet surfaces (MPS) can in some circumstances lead to elevated cladding stresses that are sufficiently high to cause cladding failure. Accurate modeling of these defects can help prevent these types of failures. The BISON nuclear fuel performance code developed at Idaho National Laboratory can be used to simulate the global thermo-mechanical fuel rod behavior, as well as the local response of regions of interest, in either 2D or 3D. In either case, a full set of models to represent the thermal and mechanical properties of the fuel, cladding and plenum gas is employed. A procedure for coupling 2D full-length fuel rod models to detailed 3D models of the region of the rod containing a MPS defect is detailed here. The global and local model each contain appropriate physics and behavior models for nuclear fuel. This procedure is demonstrated on a simulation of a boiling water reactor (BWR) fuel rod containing a pellet with an MPS defect, subjected to a variety of transient events, including a control blade withdrawal and a ramp to high power. The importance of modeling the local defect using a 3D model is highlighted by comparing 3D and 2D representations of the defective pellet region. Parametric studies demonstrate the effects of the choice of gaseous swelling model and of the depth and geometry of the MPS defect on the response of the cladding adjacent to the defect.
\end{abstract}

Keywords: multidimensional nuclear fuel behavior, multiphysics, BISON

\section{Introduction}

The cladding of light water reactor fuel rods serves as a first barrier against fission product release. Consequently, it is important to understand the causes of failure, and design and limit the conditions imposed on fuel to prevent such failure. Fuel failures are generally categorized according to four known mechanisms: 1) pellet-cladding interaction (PCI), 2) crud-induced localized corrosion, 3) debris fretting and 4) undetected defects during manufacturing [1].

PCI is largely associated with the stress-corrosion cracking mechanism that has been known to exist in $\mathrm{Zr}$-based alloys since the 1960s [2]. In a comprehensive review, Cox [3] identified four factors simultaneously necessary to produce PCI, which include stress, time, susceptible material, and chemical environment. Armed with this knowledge, fuel manufactures have worked for decades to reduce PCI related fuel failure rates. For example, Potts [4] outlines a series of improvements made to boiling water reactor (BWR) fuel (addition of a zirconium liner, pellet geometry changes, modified clad heat treatment, improved tube flaw detection) that resulted in substantial reductions in failure rates. However, even with these countermeasures, during 2003-2006 PCI resulted in approximately $12 \%$ of the clad failures (world-wide) in BWR fuel [5].

The likelihood of PCI failure can increase significantly in cases where undetected manufacturing defects are present in the

\footnotetext{
*Corresponding author

Email address: Benjamin.Spencer@inl .gov (B. W. Spencer)
}

fuel pellets. As early as 1997, such defects, known as missing pellet surface (MPS), were identified as a primary cause of clad failure [4]. Clear evidence of clad fractures adjacent to MPS defects have been observed in both BWR fuel [1,6] and, to a lesser extent, in PWR fuel [7]. Such failures are known to occur during power ramps. In one well-documented case, BWR fuel operating in a control cell failed during control blade withdrawal where it is estimated that the local power rapidly increased from roughly 15 to $35 \mathrm{~kW} / \mathrm{m}$ at the failure location [1]. Failures during removal of axial power shaping rods in PWRs are also suspected to result from local power increases in the vicinity of MPS defects [8]. Accurate modeling of MPS defects using fuel performance codes can provide better understanding of and help prevent these types of failures.

Nuclear fuel performance codes commonly use a 1.5D (axisymmetric, axially-stacked, one-dimensional radial) [9-11] or 2D axisymmetric [12] representation of the fuel rod. The behavior of the fuel and cladding in the region of an MPS defect is driven by the departure of the fuel geometry from axisymmetry. Hence, an axisymmetric representation cannot be used in the region of the defect to capture the local effects of that defect. $2 \mathrm{D}$ cross-section models in a Cartesian coordinate system have been employed to represent the defect $[13,14]$, but they have the significant drawback that any irregularity in the pellet geometry is extruded along the full length of the rod in this representation. To accurately capture the behavior of the defective pellet, which is a 3D problem, it is best to employ a 3D model. Efforts to capture 3D MPS behavior have been reported using commer- 
cial finite-element based thermomechanics tools [7, 15].

To accurately model the effects of defective pellets, it is also important to employ appropriate physics models for all aspects of the fuel behavior in the region of the defective pellet, as well as for the rest of the rod. To accomplish this, it is essential to use a 3D-capable fuel simulation code, employing applicable models for thermal and mechanical behavior of fuel and cladding and their interactions.

In addition, to represent the region in the vicinity of the defective pellet, it is also important to represent the response of the remainder of the fuel rod, and correctly account for interactions between the pellet region and the remainder of the rod. It would be possible to model the entire fuel rod in $3 \mathrm{D}$, including the geometry of the defect, although this would be very computationally expensive, and with little benefit, because the majority of the model is axisymmetric. The authors previously used a 3D model of a short rodlet with a scaled-down plenum, and containing a defective pellet $[16,17]$. By modeling a section of the rod in $3 \mathrm{D}$, this approach avoids errors potentially induced by mapping results between a global and a local model. In that approach, however, there is an inherent assumption that the entire rod experiences the same irradiation history. The most important error introduced by that assumption is that it misrepresents the fraction of fission gas in the plenum, because it assumes that the fission gas released per unit length of rod is the same throughout the rod as it is in the region of the defective pellet.

This paper presents a modeling approach that couples a $2 \mathrm{D}$ axisymmetric model of a full fuel rod with a $3 \mathrm{D}$ model of the region of a defective pellet. Both of these models are run using a fuel performance simulation code, so important physics are captured in both the global model and in the detailed model of the MPS defect region. This avoids the computational expense and limited utility of a full 3D simulation while capturing the important 3D physics in the region of the MPS defect. The primary purpose of the $2 \mathrm{D}$ full-rod model in this approach is to calculate the following quantities related to the plenum gas at each time step: fission gas released, plenum volume, and temperature. This approach is applied here to simulate a defect in a BWR fuel rod subjected to a sudden power increase due to a control rod blade withdrawal and to a high power ramp well beyond the normal operating power.

Significant improvements have been made to earlier models MPS defects in BISON [16]. The present work employs a much improved mechanism for coupling the full rod response with that of the defective pellet region, which permits realistic modeling of a variety of transients, including those of interest for BWR reactors. In addition, the current model includes an improved fission gas model, includes the combined effects of creep and plasticity, and takes advantage of significant algorithmic improvements to the BISON code, especially for contact enforcement. There are still some important effects that are not considered in the presently-employed models (e.g. fuel creep and fracture, and frictional contact), which should be included for an industrial application. However, the modeling approach is sufficiently realistic to gain understanding of the relative effects of various parameters on the cladding response due to an MPS defect. This paper presents parametric studies of the ef- fects of model dimensionality, gaseous swelling model, and of the depth and geometry of the MPS defect.

This paper is organized as follows: Section 2 provides a brief overview of the fuel performance code used in this work. Section 3 describes the models used in this work, including the discretized 2D axisymmetric full-rod and 3D MPS defect region models, and a 2D cross-section model of the defect region for comparison. Section 3 also describes the physical behavior models employed in simulations using those models. Section 4 describes the power ramp scenarios imposed on the BWR rods simulated here. Section 5 presents simulation results for a baseline scenario, while Sections 6, 7, 8, and 9 present the results of parametric studies performed using this model. Finally, Section 10 provides a summary of this work.

\section{BISON Fuel Performance Code}

The BISON fuel performance code $[16,18]$ developed at Idaho National Laboratory (INL) is used for all the simulations presented here. BISON is built on the Multiphysics ObjectOriented Simulation Environment (MOOSE) [19], also developed at INL. MOOSE is a parallel finite element framework for implicitly solving tightly coupled sets of partial differential equations using the Jacobian-Free Newton Krylov method. MOOSE supports 1D, 2D, and 3D models, and its architecture facilitates adding new material models and coupled physics.

BISON uses the MOOSE framework to solve the coupled equations relevant to fuel performance. It can solve the heat conduction, species diffusion, and solid mechanics equations in a tightly coupled manner, and provides constitutive and behavior models that are dependent on those physics. Its thermal conductivity models are dependent on temperature, porosity, and burnup. Models are provided for swelling, densification, and creep due to temperature and irradiation. Relocation and smeared cracking models account for fracture. Fission gas production and release are also modeled.

BISON employs either a 2D axisymmetric, 2D Cartesian (plane strain or generalized plane strain), or 3D representation of the fuel geometry. Finite elements using either a linear or quadratic interpolation of the field variables can be used. A master-slave contact algorithm is used for enforcing mechanical and thermal contact between the fuel pellets and the cladding.

\section{BWR fuel rod simulation models}

As described previously, the modeling approach employed here is to model the global response of the full fuel rod using a 2D axisymmetric model, and then perform a one-way transfer of relevant quantities to a detailed 3D model of the region in the vicinity of the defect. The details of the full rod geometry and the defective pellet, as well as the discretized models and modeling procedure used to represent their response are described here. 


\subsection{Two dimensional full rod model}

A 2D axisymmetric BISON model was employed to simulate the global response of an idealized BWR fuel rod. The fuel rod modeled consists of a stack of 355 fuel pellets, represented in a smeared fashion as a single continuous cylinder, encased in Zircaloy-2 cladding. Each pellet has a height of $10.35 \mathrm{~mm}$ and a diameter of $8.62 \mathrm{~mm}$, resulting in an overall fuel stack length of $3.674 \mathrm{~m}$. The cladding has an inner diameter of $8.78 \mathrm{~mm}$ and an outer diameter of $10.0 \mathrm{~mm}$. The initial radial gap between the pellets and cladding is $80 \mu \mathrm{m}$, and the height of the plenum is initially $0.25 \mathrm{~m}$.

A uniform finite element mesh is used for both the fuel and cladding along the full length of the rod. Figure 1(a) shows the full fuel rod mesh, with the axial dimension scaled by $1 / 20$ to allow for the full rod to be shown, and Figure 1(b) shows a detailed view of just the bottom portion of the mesh with no axial scaling. The mesh has 10 radial elements through the thickness of the fuel and 5 radial elements through the thickness of the clad. Quadratic 8-noded quadrilateral elements are used throughout the model. The mesh contains a total of approximately 35000 nodes and 10000 elements.

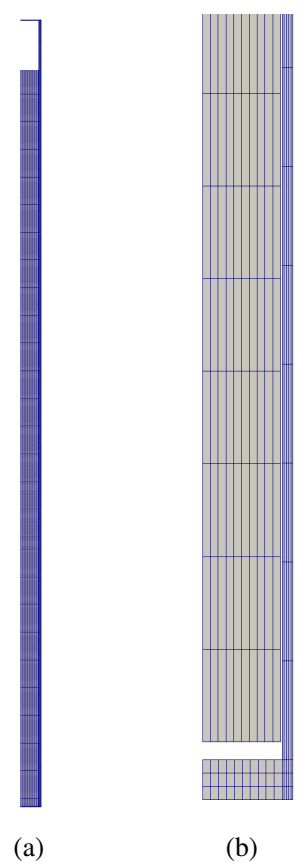

Figure 1: Finite element mesh of full rod. (a) Axisymmetric 2D model of entire fuel rod, with axial dimension scaled by $1 / 20$ (b) Detailed view of bottom of $2 \mathrm{D}$ axisymmetric fuel rod model with no axial scaling.

\subsection{Three dimensional model of defect region}

The 3D model employed to represent the local effects of the MPS defect on the fuel and cladding represents all geometric aspects of the fuel pellets, including dishes, chamfers, and defects. Figure 2 shows the geometry of a pellet with the baseline type of defect represented in most of the analyses presented here. A constant-depth slice is removed from one side of the pellet. This is a simplified representation of the type of damage that could occur due to accidental machining of the side of the pellet. Meshes with varying defect depth are employed to study the effect of defect depth on the solution. (a)

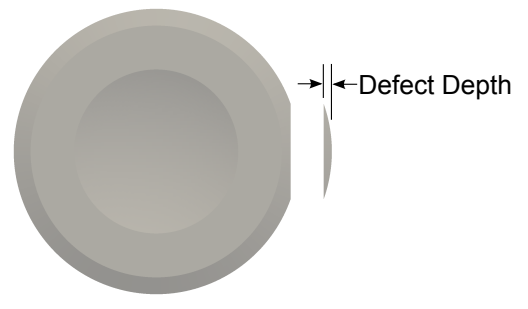

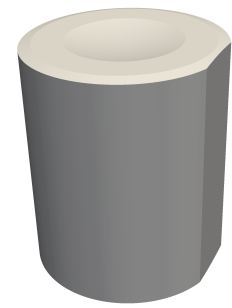

(b)
Figure 2: Geometry of defective pellet modeled in current study with dish, chamfers, and missing surface defect. (a) View from one end, showing material removed by defect (b) Projection showing full pellet with missing surface defect.

The 3D finite element mesh shown in Figures 3(a) and 3(b) is used to model the defective pellet region in BISON. The model takes advantage of two symmetry planes, both passing through the center of the defect in orthogonal orientations. Appropriate boundary conditions are applied to enforce symmetry conditions on those planes. 27-noded quadratic elements are used for both the fuel and the cladding because of their ability to represent 3D curved geometry of the outer fuel surface and inner cladding smoothly for thermal and mechanical contact. This model has approximately 140000 nodes and 16000 elements. There are 10 elements across the radial direction in the fuel, and 5 elements through the thickness of the cladding.

The response of the cladding in the vicinity of the MPS defect is of primary interest in this model. The contact algorithm employed here requires that similar mesh density be used for both the fuel and cladding, so the mesh density in that region of interest drives the mesh density of the entire model. The second order 27-noded hexahedral elements give a higher order representation of the solution, which allows the use of a coarser mesh than would be required with linear elements. The mesh density in the radial direction of the cladding is more than adequate, but ideally, a mesh density higher than that employed here should be used in the axial and circumferential directions of the cladding. Refinement in those directions drastically increases the size of this 3D model, so the current choice of mesh size is driven by computational resource considerations. Local refinement in the vicinity of the defect could be used in the future for improved solution accuracy in this region of interest.

In this model, the cladding extends beyond the end of the pellets to ensure that the fuel never slides past the end of the cladding as it expands. The results in the pellet nearest to the end are inaccurate due to end effects. Accurately representing the response of the defective pellet region requires that at least one pellet adjacent to the defective pellet that is not subject to end effects be included, so two standard pellets are included in addition to the defective pellet.

In all of the models employed in this study, thermal and mechanical contact are enforced between the fuel and cladding. Because a discrete representation of the individual fuel pellets 
is employed in the 3D models, appropriate thermal and mechanical interactions between neighboring fuel pellets must also be incorporated in the model. The rings of nodes around the outside of the dished regions on the top and bottom of neighboring pellets are merged together to mechanically connect the neighboring pellets, but still permit unrestrained deformation of those surfaces. This is a done in lieu of enforcing mechanical contact constraints between the pellets, which would be much more computationally challenging, and would have a minor effect on these results since relative sliding would be negligible under the conditions considered here. Thermal contact is enforced between neighboring pellets. This is important to permit heat transfer from the defective pellet, which has elevated temperatures, to its neighboring pellets.

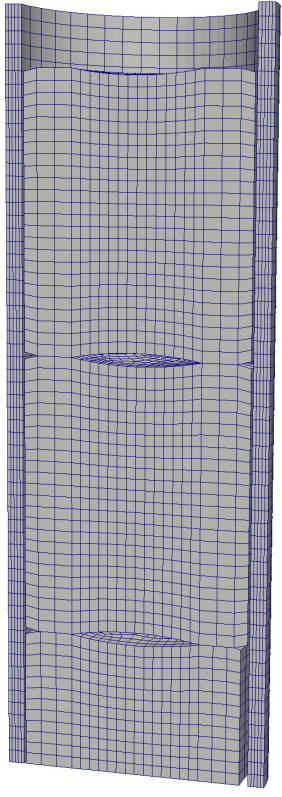

(a)

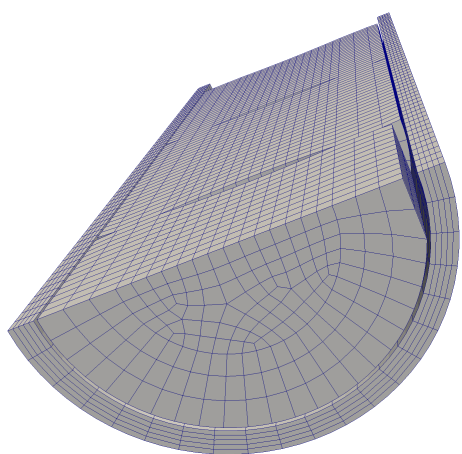

(b)
Figure 3: Two views of baseline 3D finite element mesh of region of defective pellet showing fuel and cladding. (a) View onto symmetry plane down axis of fuel rod. Defect is on right hand side of bottom pellet cut by symmetry plane (b) View onto intersection of two symmetry planes, showing details of defective pellet.

Cladding for BWR fuel rods typically includes a thin ductile zirconium liner on the inner surface. To incorporate a liner would require a mesh resolution finer than that used for the local models of the defect region employed here. The liner could be included with appropriate modifications to the mesh and appropriate nonlinear material models for the liner material. The liner has not been included in the current work, but will be included in the future as appropriate material models are developed in BISON.

\subsection{Two dimensional model of defect cross-section}

To demonstrate the importance of employing a 3D representation of the defective pellet, a 2D finite element model is also used to represent the defective pellet region for comparison. This model uses a generalized plane strain representation of a slice normal to the fuel rod axis through the defective pellet, as shown in Figure 4. The finite element mesh is identical to the mesh in the 3D model on the symmetry plane surface, except that it is mirrored about the symmetry plane passing through the center of the defect in the 3D model. The mesh uses 9-noded quadratic elements for both fuel and cladding. The model has approximately 1200 nodes and 300 elements. As for the 3D model, this model employs a symmetry plane through the defect, with appropriate symmetry boundary conditions.

Expansion and contraction along the direction of the fuel rod axis (the out-of-plane direction in the 2D model) are permitted, but two independent constraints are imposed on this outof-plane strain: one enforcing it to be constant everywhere in the fuel, and another enforcing it to be constant everywhere in the cladding. In other words, planar sections of the fuel and cladding are independently forced to remain planar. These independent constraints mimic the behavior of the frictionless contact employed between the fuel and cladding in the 3D model of the defect region, which permits unrestrained relative motion between the two bodies.

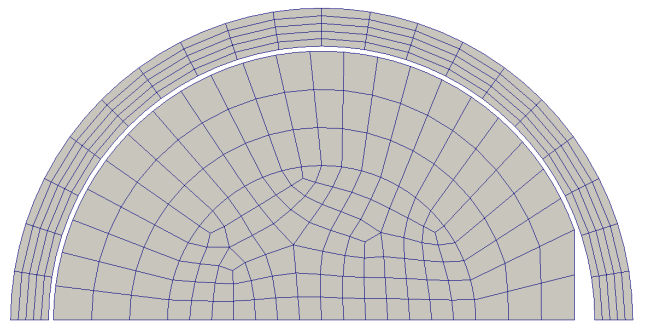

Figure 4: 2D finite element model of cross-section of defective pellet.

\subsection{Physical behavior models}

The primary objective of the present work is to quantify the mechanical response of the cladding in the region adjacent to the defective pellet. To that end, BISON is used to solve coupled heat conduction and solid mechanics equations with the discretizations described above, and appropriate models are employed to represent the behavior of the fuel, cladding, and gap/plenum, which collectively comprise the fuel rod system.

Power is applied using time histories of linear power and axial power factors to simulate BWR operating conditions. The details of these are described in Section 4. The full rod simulation incorporates computations of the radially varying power and burnup for $4.5 \%$ enriched $\mathrm{UO}_{2}$ fuel on an auxiliary mesh with approximately 14000 nodes, using the approach of [20]. Because of the complexities involved in computing the radial power in the presence of the MPS defect, the local models do not include any radial variation in the power. Incorporating a radial power profile with defective pellets is a topic of future research.

The $\mathrm{UO}_{2}$ pellets have temperature and burnup-dependent thermal conductivity, as described in [16]. A linear elastic model is employed to represent the mechanical constitutive behavior of the fuel, with a Young's modulus of $200 \mathrm{GPa}$ and Poisson's 
ratio of 0.345 . The ESCORE relocation model [12] is used to represent radial relocation of the fuel. Volumetric changes due to thermal expansion, gaseous and solid swelling, and densification are incorporated. A thermal expansion is incorporated using a constant expansion coefficient of $10 \times 10^{-6} \mathrm{~K}^{-1}$.

Burnup-dependent fuel solid swelling is incorporated using the empirical correlation from MATPRO [21]

$$
\Delta \epsilon_{s w-s}=5.577 \times 10^{-5} \rho \Delta B u
$$

where $\Delta \epsilon_{s w-s}$ is the volumetric solid swelling increment, $\Delta B u$ the burnup increment (fissions/atoms- $\mathrm{UO}_{2}$ ), and $\rho$ is the density $\left(\mathrm{kg} / \mathrm{m}^{3}\right)$. The ESCORE [12] model is employed for densification.

Modeling of fuel swelling due to fission gas bubbles is highly important for simulations of pellet-clad mechanical interaction (PCMI) under power ramp conditions, as gaseous swelling significantly contributes to the fuel radial expansion, and hence, PCMI [22, 23]. The accurate calculation of gaseous swelling during off-normal conditions such as power ramps calls for the use of physics-based models rather than empirical correlations, because of their limited range of applicability. In the present work, a physics-based model is adopted for the concurrent calculation of gaseous swelling and fission gas release. This model incorporates the underlying physical mechanisms of gas generation, diffusion, bubble growth and inter-connection, and accounts for the inherent coupling between gaseous swelling and fission gas release (FGR). Model assumptions and equations are detailed in $[24,25]$. In this paper, this physically based gaseous swelling model is referred to as the Coupled model, as it directly considers the inherent coupling with fission gas release, in contrast to empirical gaseous swelling models. The following empirical gaseous swelling model from MATPRO [21] is also available in BISON, and is used in the present work for comparison with the Coupled model:

$$
\begin{aligned}
& \Delta \epsilon_{s w-g}=1.96 \times 10^{-31} \rho \Delta B u(2800-T)^{11.73} \\
& * e^{-0.0162(2800-T)} e^{-0.0178 \rho B u}
\end{aligned}
$$

where $\Delta \epsilon_{s w-g}$ is the volumetric gaseous swelling increment, $B u$ and $\Delta B u$ are the burnup and burnup increment (fissions/atoms$\mathrm{U})$, respectively, $\rho$ is the density $\left(\mathrm{kg} / \mathrm{m}^{3}\right)$ and $T$ is the temperature $(\mathrm{K})$. This empirical gaseous swelling model is referred to here as the MATPRO model. The Coupled model is used for all models presented here except for where it is specifically noted that the MATPRO model is used for comparison with that model.

The elastic model employed here for the fuel mechanical behavior neglects the effects of fracture and creep. These can potentially have a significant effect on cladding response during PCMI, and will be incorporated in future simulations of this type as BISON's fracture models are further developed.

The cladding material is assumed to be Zircaloy- 2 with a recrystallized annealed (RXA) microstructure. The thermal conductivity and thermal expansion coefficient are assumed constant at $16 \mathrm{~W} / \mathrm{m} / \mathrm{K}$ and $5 \times 10^{-6} \mathrm{~K}^{-1}$, respectively. Elastic mechanical behavior is modeled using a Young's modulus of 75
GPa and Poisson's ratio of 0.3 . Plasticity is governed by a combined creep and time-independent plasticity model, as described below.

Creep is based on the model of Limbäck [26] and includes both thermal and irradiation behavior. Thermal creep is given by the Matsuo correlation [27] with the creep rate $(1 / \mathrm{h})$ given by

$$
\begin{aligned}
\dot{\epsilon}_{t h} & =A \frac{E}{T}\left(\sinh \frac{a_{i} \sigma_{m}}{E}\right)^{n} e^{-Q / R T} \\
a_{i} & =a\left[1-A_{1}\left(1-e^{\left(-A_{2} \Phi^{A_{3}}\right)}\right)\right] \\
E & =1.148 \times 10^{5}-59.9 T
\end{aligned}
$$

where $T$ is temperature $(\mathrm{K}), \Phi$ is the fast neutron $(>1 \mathrm{MeV})$ fluence $\left(\mathrm{n} / \mathrm{m}^{2}\right), \sigma_{m}$ is the effective (Mises) stress (MPa), $Q$ is the creep activation energy $(198 \mathrm{~kJ} / \mathrm{mol}), R$ is the gas constant $(8.314 \mathrm{~J} / \mathrm{mol} / \mathrm{K}), A=5.47 \times 10^{8} \mathrm{~K} / \mathrm{MPa} / \mathrm{hr}, A_{2}=1.4 \times 10^{-27}$ $\left(\mathrm{n} / \mathrm{cm}^{2}\right)^{-A_{3}}$ and $a, n, A_{1}$ and $A_{3}$ are dimensionless constants given by $650,3.5,0.56$ and 1.3 , respectively. The irradiationinduced creep rate $(1 / \mathrm{hr})$ is given by the empirical model of Hoppe [28]

$$
\dot{\epsilon}_{i r}=C_{0} \phi^{C_{1}} \sigma_{m}{ }^{C_{2}}
$$

where $\phi$ is the fast neutron $(>1 \mathrm{MeV})$ flux $\left(\mathrm{n} / \mathrm{m}^{2} \mathrm{~s}\right), \sigma_{m}$ the effective (Mises) stress (MPa), and $C_{0}, C_{1}$, and $C_{2}$ are material constants. For the clad alloy assumed here, $C_{0}=1.654 \times 10^{-24}$ $\left(\mathrm{n} / \mathrm{m}^{2} / \mathrm{s}\right)^{-C_{1}}(\mathrm{MPa})^{-C_{2}} / \mathrm{hr}$ and $C_{1}$ and $C_{2}$ are dimensionless constants given by 0.85 and 1 , respectively. Note that the original Hoppe formulation is given in terms of circumferential stress, whereas the relation implemented in BISON assumes an effective (Mises) stress. The secondary creep rate is then

$$
\dot{\epsilon}_{s}=\dot{\epsilon}_{t h}+\dot{\epsilon}_{i r}
$$

Primary creep is defined as

$$
\begin{aligned}
& \epsilon_{p}=\epsilon_{p}^{s}\left(1-e^{\left(-C \sqrt{\dot{\epsilon}_{s} t}\right)}\right) \\
& \epsilon_{p}^{s}=B \dot{\epsilon}_{s}^{b}\left[2-\tanh \left(D \dot{\epsilon}_{s}\right)\right]^{d}
\end{aligned}
$$

where $B=0.0216\left(\mathrm{hr}^{b}\right), D=35500 \mathrm{hr}$ and $C, b$, and $d$ are dimensionless constants of 52, 0.109 and -2.05 , respectively. Total creep strain is the combination of the primary and secondary creep:

$$
\epsilon_{c}=\epsilon_{p}+\dot{\epsilon}_{s} t
$$

Time-independent plasticity is modeled using $J_{2}$ plasticity with linear strain hardening. For the cladding material considered here, a yield strength of $550 \mathrm{MPa}$ and plastic modulus of $2.5 \mathrm{GPa}$ was assumed. For each stress update, the model first considers only the creep contribution to compute a new stress, which is then compared to the yield strength. If that stress is above the current yield stress, instantaneous plasticity is also applied to reduce the stress to the current yield stress. Iterations are employed until both the creep and plasticity models are converged.

Mechanical and thermal interactions between the cladding 
and fuel stack are represented using contact algorithms. These consist of a geometric search phase, in which nodes on the slave surface are paired with the element faces on the master surface onto which they are currently projected, and an enforcement phase. In the enforcement phase, strict non-penetration constraints are imposed on slave nodes that would have otherwise penetrated the master surface. In the current work, frictionless interactions are employed. Frictional interactions between the fuel and pellet can have an important effect on the cladding mechanical response in pellet-clad mechanical interactions, and including frictional effects is the subject of ongoing development. The conductance across the fuel/cladding gap used in thermal contact enforcement is based on distance, roughness, gas composition, and surface emissivity, as described in detail in [16].

\subsection{Initial and boundary conditions}

The initial plenum gas consists of He pressurized to $290 \mathrm{kPa}$, and the external coolant exerts a pressure of 7.24 MPa on the outside of the rod during operation.

A flux boundary condition is used to model heat transfer from the cladding to the coolant. This boundary condition employs a constant convection coefficient of $100 \mathrm{~kW} / \mathrm{m}^{2} / \mathrm{K}$ to calculate the flux between the cladding surface, which has a calculated temperature, and the fluid, which has a prescribed temperature of $561 \mathrm{~K}$.

\subsection{Coupling between full rod model and defect region model}

In the modeling approach used in the present work, the 2D full rod model is used to calculate the response of the fuel rod, with the assumption that local effects of the defective pellet have a negligible effect on the full rod response. That assumption permits the models to be run in a one-way coupled manner, in which the full rod model is first run to completion, after which time histories of results from that model are applied as boundary conditions to the 3D model of the defective pellet region. Both the full rod model and defective pellet region models are run through the same full irradiation history.

In the present work, the only results passed from the global to local model are those related to the plenum gas. Specifically, the transferred quantities are the time history of the total fission gas released, total plenum volume, and plenum gas temperature. Using an ideal gas law, these three quantities are used, together with the initial He fill gas pressure, to calculate the plenum pressure and plenum gas composition at each time in the analysis. Because the plenum volume is essential to calculate the plenum gas behavior, fuel performance models typically represent the plenum as a closed volume. As can be seen in Figure 3, the defective pellet model employed here does not have a closed plenum because that is not necessary. The plenum pressure is directly calculated from the quantities transferred from the full rod model, and applied as a pressure boundary condition to the inner surface of the fuel rod.

The present approach does not account for the change in fission gas released due to local differences in the behavior in the defective pellet region, and also assumes that pressure and gas composition are uniform throughout the fuel rod. These are reasonable assumptions for most conditions.

\section{Simulation of power ramps in BWR rods}

The approach detailed in the preceding sections has been applied to simulate the local response of fuel and cladding in the vicinity of a MPS defect as that region of the fuel rod is subjected to power ramps. Two types of power ramp scenarios are simulated here: the rapid local power increase in fuel rods adjacent to a control blade due to a withdrawal of that control blade, and a ramp of the overall rod power to a significantly higher level, followed by holding the power at that level for a sustained period of time.

If a fuel rod is located in a position adjacent to a control blade, the power is suppressed in that rod due to effects of the control blade. When it is necessary to adjust the position of control blades, they are inserted or withdrawn in small increments known as "notches". Although an incremental control blade withdrawal has a relatively minor impact on the average linear power of the entire fuel rod, the section of the fuel rod located slightly below the tip of the control blade experiences a rapid increase in power because of the resulting increase of thermal neutrons. This type of sudden power increase could result in significantly elevated stresses in the presence of a fuel defect. The event takes place quickly enough that creep mechanisms have insufficient time to relieve stresses.

The effects of a ramp of the power over the entire reactor would be different than a local power increase resulting from a blade pull. In this case, the power is ramped up over a longer period of time, so there could be more opportunity for creep to relax stresses caused by pellet-clad mechanical interactions. If the whole rod experienced a significant ramp in power beyond a baseline level, there could also be a significant fission gas release, causing elevated fuel temperatures due to decreased gap conductance. To demonstrate the ability of the proposed modeling technique to capture the effects of both of these types of power ramp, a simulated rod is subjected to an idealized irradiation history in which representative events of both types occur.

In this idealized history, the rod is initially irradiated at a constant power in a position away from a control blade for a two-year operating cycle, after which it is moved into a position adjacent to a control blade that is inserted about $50 \%$ into the reactor. The defective pellet is axially located slightly below the tip of that control blade, so the power level at the defective pellet decreases significantly from its previous level at the beginning of the second operating cycle after the rod containing the defect is moved. During the second cycle, the control blade is withdrawn, resulting in a rapid power ramp at the location of the defective pellet. Following this event, the overall power and control blade position are held constant for a significant period of time, allowing creep mechanisms to relieve elevated stresses in the cladding. Following this period, the overall power is then ramped up to a level $50 \%$ higher than the baseline power and held at that level.

The power is provided to the BISON models using the product of a time-dependent linear heat generation rate (LHGR) and a time-dependent axial power profile. The power history is provided in tabular form in Table 1, with descriptions of the events at various times and alphabetical identifiers for those events, 
Table 1: Time history of power in tabular form with descriptions and alphabetic identifiers for events. Power is linearly interpolated in time between the points listed here. This history is provided in terms of power factors applied to a baseline linear power of $25 \mathrm{~kW} / \mathrm{m}$.

\begin{tabular}{lrrll} 
Id & Time (s) & Time (d) & $\begin{array}{l}\text { Power } \\
\text { factor }\end{array}$ & Description \\
\hline $\mathrm{a}$ & 0 & 0 & 0.0 & Begin ramp up \\
$\mathrm{b}$ & 43200 & 0.5 & 1.0 & At base power \\
$\mathrm{c}$ & 63062000 & 729.88426 & 1.0 & Begin ramp down \\
$\mathrm{d}$ & 63072000 & 730.00000 & 0.0 & Refuel, shuffle \\
$\mathrm{e}$ & 63115000 & 730.49769 & 1.0 & At base power \\
$\mathrm{f}$ & 71000000 & 821.75926 & 1.0 & Begin ramp down \\
$\mathrm{g}$ & 71000100 & 821.76042 & 0.9 & At lower power \\
$\mathrm{h}$ & 71000110 & 821.76053 & 0.9 & Blade pull \\
$\mathrm{i}$ & 71001900 & 821.78125 & 0.9 & Begin ramp up \\
$\mathrm{j}$ & 71005500 & 821.82292 & 1.0 & At base power \\
$\mathrm{k}$ & 72000000 & 833.33333 & 1.0 & Begin ramp up \\
$\mathrm{l}$ & 72010000 & 833.44907 & 1.5 & At high power \\
$\mathrm{m}$ & 72060000 & 834.02778 & 1.5 & Hold power
\end{tabular}

which will be referenced in the presentation of results for those times. This power history also plotted graphically in the upper pane of Figure 7. The LHGR for the first irradiation cycle of 2 years is held constant at $25 \mathrm{~kW} / \mathrm{m}$ throughout the irradiation. The power is then ramped down to zero over a few hours before being ramped back up to $25 \mathrm{~kW} / \mathrm{m}$ over $12 \mathrm{~h}$. About 91 days into this second cycle, the control blade withdrawal is initiated. For this procedure, the power is first ramped down to $90 \%$ of the base LHGR to reduce the effect of the large power ramp that occurs in fuel pellets near to the end of the blade. The blade is withdrawn two notches, corresponding to $179 \mathrm{~mm}$ of movement. The blade movement occurs within a few seconds. After the blade is moved, the overall power level is kept at $90 \%$ for approximately 30 minutes and is then slowly ramped back up to full power over the next hour.

The axial peaking factors used in this work are shown in Figure 5. The axial profile during the initial 2-year irradiation period is a truncated cosine to approximate the conditions of a fuel rod located distant from any control blades. At the beginning of the second irradiation, the axial profile of the rod power is adjusted to reflect the movement of the rod to a position adjacent to a partially inserted control blade. The fission rate in the portion of the rod shielded by the blade is axially uniform and is approximated to be $1 / 3$ of the peak power in the rod. The axial profile of the fission rate in the portion of the rod above the blade matches the truncated cosine used in the first cycle. The power is ramped up from the lower power level used to represent the shielded portion of the rod to the truncated cosine profile over a short distance near the control blade tip. The control blade withdrawal is simulated by shifting the axial position of the transition from the flat, low power profile to the truncated profile. The axial profiles before and after the control blade withdrawal are shown in Figure 5.

The power history applied to the local model of the defective pellet region is determined using the peaking factor function used in the global model evaluated at the axial location of the de- fective pellet. For simplicity, the axial power is not varied over the local model, which represents a small portion of the overall rod. The defective pellet is assumed to be located at a position $1.75 \mathrm{~m}$ from the bottom of the rod, which is just below the control blade tip before the blade pull event, and just above it after that event. As a result, the axial peaking factor for that location instantaneously jumps from 0.34 to 0.99 during the blade pull.

The axial profiles used here are simply idealizations of actual conditions. In reality, the axial profile in a BWR would be biased rather than symmetric, and the region immediately above the tip of the control blade would be elevated after a control blade withdrawal because there would be a lower buildup of reaction-poisoning fission products in that region as a result of the decreased power experienced in that region prior to the blade withdrawal. Applying more realistic conditions informed by neutronic and thermal-hydraulic calculations would improve the accuracy of the simulations, but the approximations used here are sufficient to demonstrate the methods, understand the mechanisms involved, and investigate sensitivities to parameters.

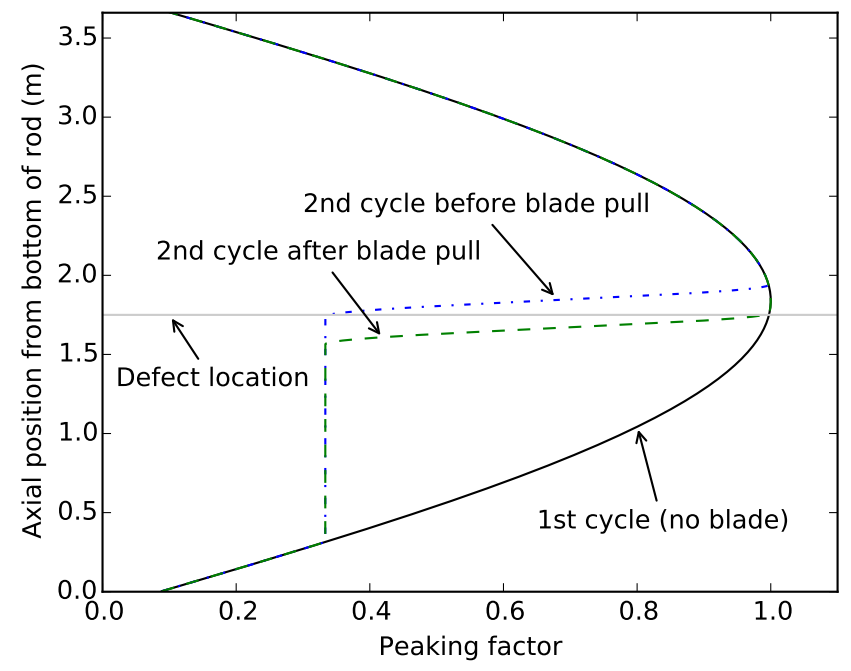

Figure 5: Axial power factors for the 2D full rod simulation. During the first irradiation period of 2 years, the rod is not located near a blade, so the axial power profile is a truncated cosine. During the second irradiation period, the rod is located close to a rod that is roughly $50 \%$ inserted. A blade pull sequence moves the blade down by $179 \mathrm{~mm}$.

\section{Simulation Results}

\subsection{Full Rod Simulation Results}

Figure 6 shows temperature contours on the 2D axisymmetric full rod model at selected points in time during the irradiation history. The horizontal line on each of these plots indicates the axial position of the defective pellet considered here. The thermal state of the rod during irradiation in a position away from a control blade, next to the control blade before and after the partial blade withdrawal, and at the peak of the final power ramp are shown. While the blade pull event has little effect on 
the global rod behavior, it is evident by comparing the results shown at times $(\mathrm{g})$ and $(\mathrm{h})$ that it results in a significant and sudden increase in temperature at the position of the defective pellet.

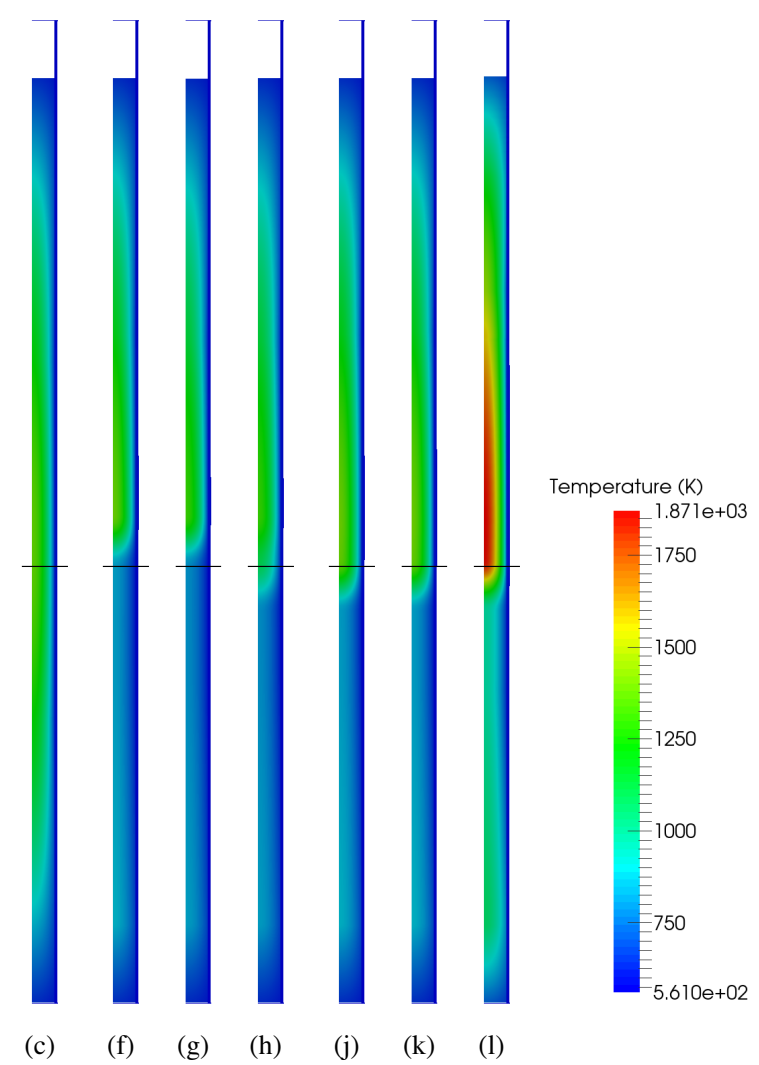

Figure 6: Temperature contours from 2D full rod model at key times in the power history. Letters in figure captions are identifiers for the solution times, as provided in Table 1. Horizontal lines indicate the axial location of the defective pellet. The axial dimension is scaled by a factor of $1 / 20$ to show the entire rod.

Figure 7 shows the time histories of the three output quantities computed by the full rod model that are transferred to the local defect models: fission gas released, average plenum gas temperature, and plenum volume, as well as the power history used as input to that model for reference. These quantities are shown for the full irradiation history, as well as during the blade pull and high power ramp events on shorter time scales to highlight the behavior during those events. Because the blade pull event only affects a small portion of the rod, it has a negligible effect on the global plenum gas properties. The $50 \%$ power ramp above the baseline power late in life, however, results in a significant increase in the fission gas released, a noticeable increase in the average plenum gas temperature, and a decrease in the plenum volume due to thermal expansion and gaseous swelling of the fuel.

Once the time histories of the plenum gas properties were obtained from the 2D full rod model, they were applied to multiple local models of the defective pellet to explore the effects of various modeling assumptions and flaw geometries on the local thermomechanical cladding response. The cladding response is presented in detail first for a baseline case in which a
3D local model is used to represent a $0.1 \mathrm{~mm}$ deep flaw. Next, to demonstrate the importance of modeling the local defect region in 3D, a comparison of the baseline 3D results with similar results for a $2 \mathrm{D}$ generalized plane strain representation of the defect is shown. The baseline response is then compared to results obtained using a different model for gaseous swelling, to demonstrate the role of a physically based treatment of this phenomenon in the simulation of power ramps. Finally, selected results of a parametric study in which the defect depth is varied are presented.

\subsection{Local Simulation Results: 3D Baseline Case}

The distance between the pellet and the clad is much greater adjacent to the MPS defect than it is for undamaged pellets. This causes a significant decrease in the conductance across the gap and a corresponding decrease in heat transfer between the pellet and clad adjacent to the defect. The temperature is thus elevated in the fuel near the surface with the defect and at the centerline. Because of the higher fuel temperature, the cladding temperature is elevated around the boundaries of the defect, where the gap conductance is unaffected by the defect. Immediately adjacent to the defect, the cladding temperature is significantly decreased because of the lower gap conductance.

These effects occur throughout the irradiation history, and can be observed in the representative temperature contours for the baseline case with a $0.1 \mathrm{~mm}$ deep defect shown in Figure 8, which are shown at the end of the analysis. Figure 8(a)shows the pellet and clad temperature, and Figure 8(b) shows just the clad. The cladding results are provided from two viewpoints to show the response on the interior and exterior of the cladding in the defect region. The displacements are magnified 10x, highlighting the cladding deformation due to PCMI. The fuel centerline temperature is modestly elevated in this case, but would be elevated much more with a deeper defect.

Figure 9 shows the mechanical effects of the MPS defect with contour plots of the hoop stress, von Mises stress, and hoop creep strain in the cladding in Figures 9(a), 9(b), and 9(c), respectively. As for the thermal results, these are shown at the end of the analysis with displacements magnified 10x. The clad across from the defect bends inward due to the coolant pressure, and is supported around the boundaries of the defect in the pellet containing the defect and on the rims of the pellets above and below the defect. These regions have high contact pressures and elevated stresses in the cladding. This region exhibits classical plate bending behavior, with high tensile stresses in the interior of the cladding and high compressive stresses on the exterior of the cladding at the center of the defect. Around the boundaries of the defect, the stresses are reversed, with high compressive stresses on the cladding interior and high tensile stresses on the cladding exterior.

Cladding creep effects the mechanical response significantly by relaxing stresses, both during the base irradiation and during the power ramp. Creep has a far more significant effect than plasticity in the conditions studied here. Plots of plastic strains are not included because they are negligible. Cladding creep is highly sensitive to temperature, which is demonstrated in these results to be affected significantly by the MPS defect. 

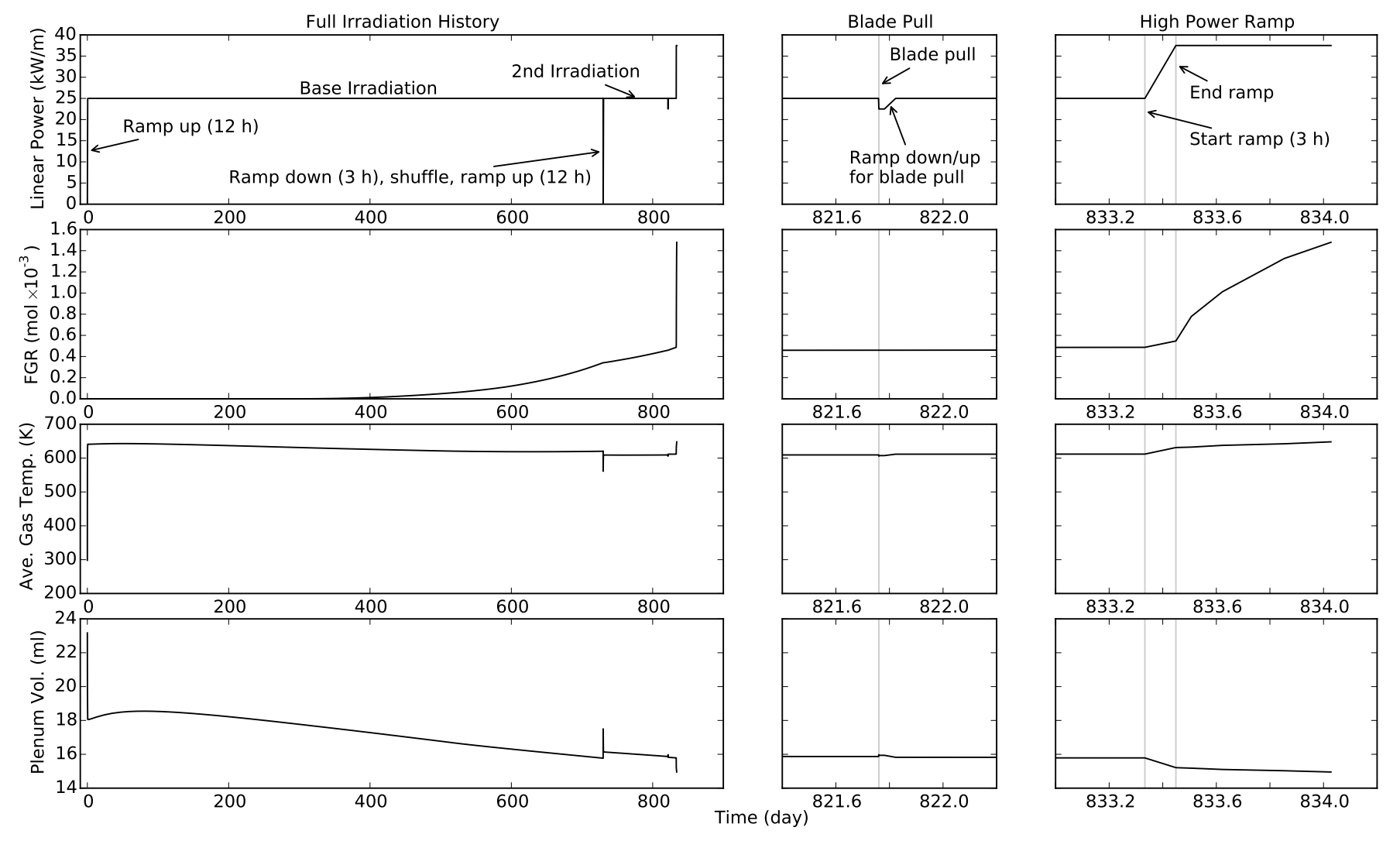

Figure 7: Time histories of linear power and output quantities of interest passed from full rod model to submodels: fission gas released (FGR), average plenum gas temperature, and plenum volume. Time histories show first cycle away from control blade, second cycle near control blade, blade pull, and high power ramp.

A series of time history plots of field quantities of interest at selected locations in the cladding adjacent to the defective pellet have been generated. Figure 10 shows a diagram of the locations (with abbreviations) for which time histories of quantities of interest are reported for both the 3D models and 2D models (which will be discussed later). Results are reported at the interior and exterior of the cladding at the waist of the defective pellet, and at the interface between the defective pellet and its neighboring, non-defective pellet. Figure 11 shows time histories of the von Mises stress, axial stress, hoop stress, hoop creep strain, and temperature during the full irradiation history at these locations for the baseline 3D model of the $0.1 \mathrm{~mm}$ deep defect. This figure shows three plots on different time scales for each of these quantities: one covering the full irradiation history, one covering the blade pull event, and a similar plot covering the high power ramp at the end of the irradiation history.

A number of observations can be made from these plots:

- The effects of pellet/cladding mechanical interaction are observed first at the pellet/pellet interface (after around 370 days), and later (around 480 days) for the waist region. This is expected because of the deformation of the pellets into an hourglass shape, which causes the top and bottom of the pellet to come in contact with the cladding before the pellet waist.

- Pronounced bending effects in the hoop direction can be observed in the plots of the hoop stresses at both the pel- let/pellet interface and the waist (in the middle of the defect region) once mechanical contact is established. At the pellet/pellet interface, the cladding is bent outward (higher tensile stresses on the exterior), while in the center of the defect, it is bent inward (higher tensile stresses on the interior).

- After the refueling event at 730 days, the power is low enough at this location to re-open a gap between the fuel and cladding, resulting in a reversal of the hoop stresses. This causes the hoop creep strains that decrease over time, rather than increase over time, as they do when there is mechanical contact between the fuel and cladding. This is an example of deconditioning, in which the cladding diameter decreases due to creep-down. This deconditioning causes the cladding to experience higher stresses during sudden power ramps from this state.

- At both the pellet/pellet interface and waist locations, the blade pull event does not result in a noticeable increase in hoop creep strains, but during and after the high power ramp, there is a significant increase in the hoop creep strain at the cladding interior. The creep strain at the cladding exterior is virtually unaffected by either of these transients because the temperatures are significantly (over $20 \mathrm{~K}$ ) lower at the exterior.

- After the high power ramp, the hoop stress at the pel- 


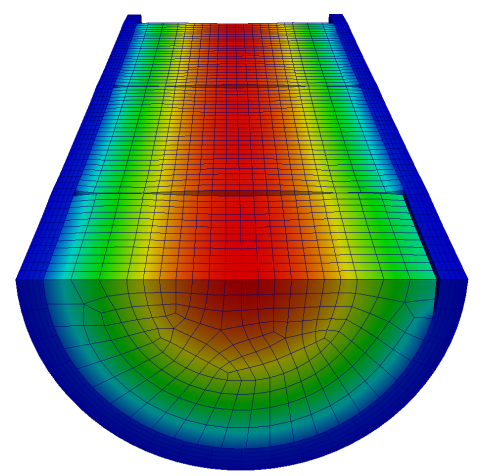

561.5706 .75 1060.1 1413.5

(a)

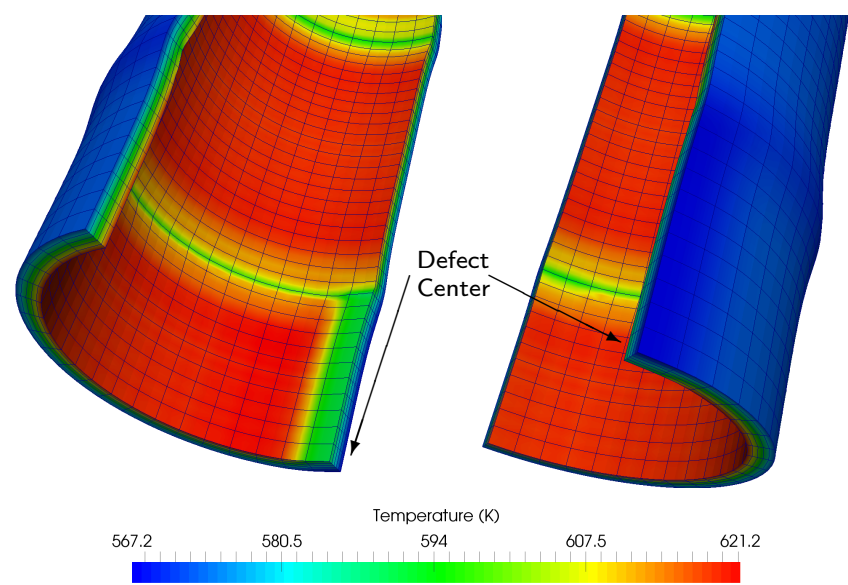

(b)

Figure 8: Temperature solution for baseline case $(0.1 \mathrm{~mm}$ deep flaw) at end of analysis (time point $\mathrm{m}$ ), for (a) fuel and cladding and (b) cladding only. Cladding results are provided from two viewpoints to show the response on the interior and exterior in the defect region. Displacements are magnified 10x for the cladding-only case.

let/pellet interface decreases noticeably due to the significant creep strains experienced at that location. At the waist, the creep strains are significantly lower, and no such decrease in hoop stress is observed. The greater hoop creep strains at the pellet/pellet interface are somewhat counter-intuitive because the hoop stress is lower at that location than at the waist, but this can be explained by the fact that the von Mises stress is higher at that the pellet/pellet interface because of the combination of high tensile hoop stress and compressive axial stress, which is caused by local bending in the axial direction at this location.

\section{Comparison of 2D Cross Section and 3D Representations of Defective Region}

The contour plots of fields of interest from the 3D simulation shown in Figures 8 and 9 and the time history plots of quantities of interest at various locations shown in Figure 11

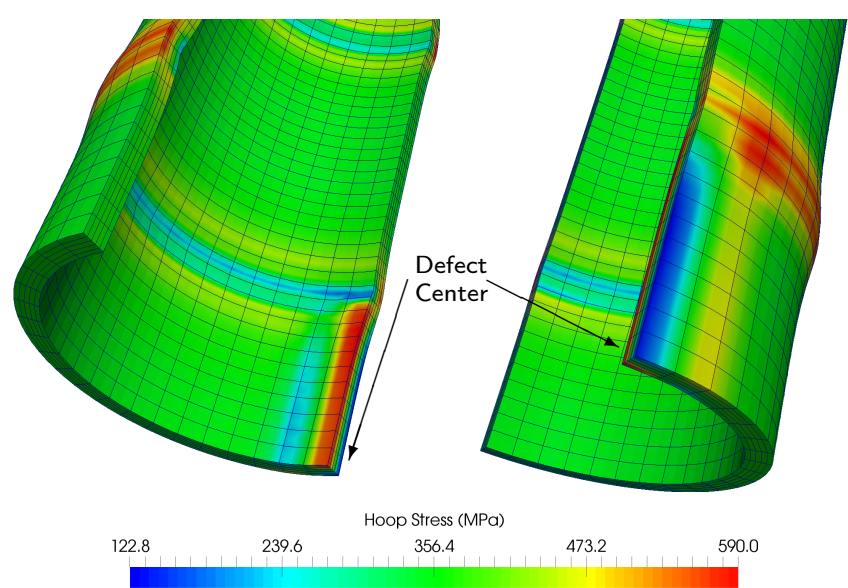

(a)

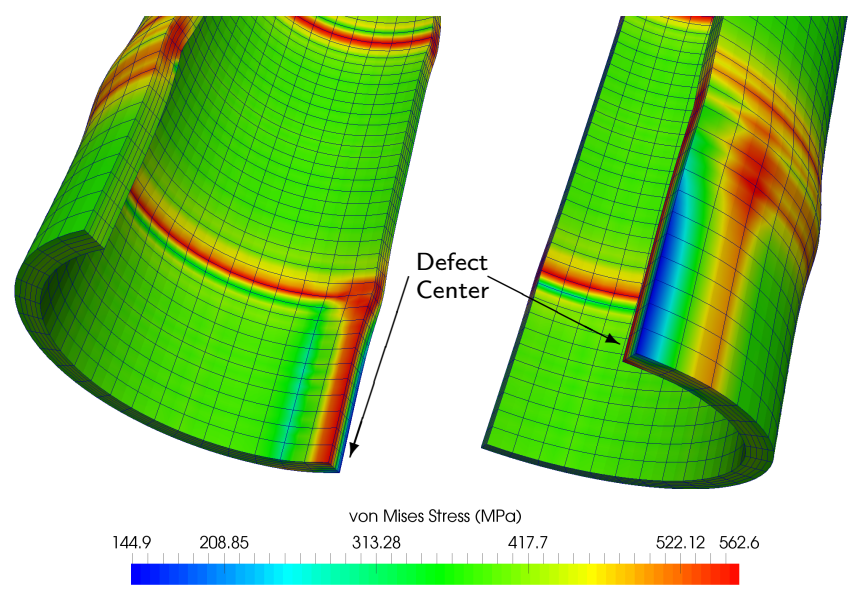

(b)

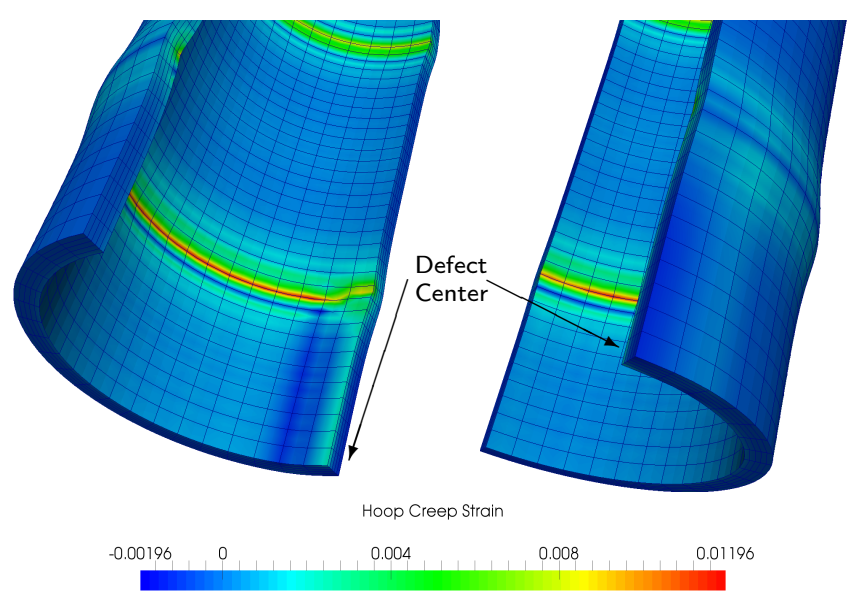

(c)

Figure 9: Cladding mechanical response for baseline case (0.1 mm deep flaw) at end of analysis (time point m), showing contours of (a) hoop stress, (b) von Mises stress and (c) hoop creep strain from two viewpoints. Displacements are magnified 10x. 


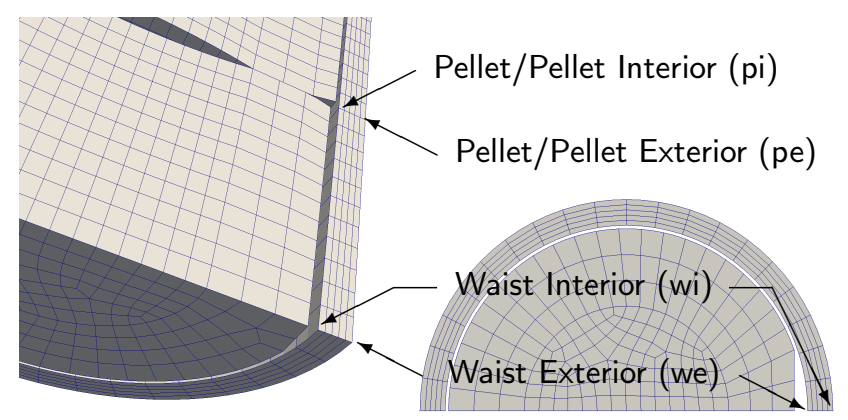

Figure 10: Locations in cladding on $3 \mathrm{D}$ and 2D cross-section models where time histories of results are reported, with abbreviations used to refer to these locations in time history plots.

clearly show that there are significant variations in the cladding response along the axial direction of the rod in the vicinity of the defect. These effects are naturally represented by the 3D model of the defective region. To further demonstrate the importance of adopting a 3D model to capture these effects, the results of the 2D model of a cross-section of the defective pellet described in Section 3.3 are presented and compared with equivalent 3D model results here.

Time histories of the response at the cladding interior for 2D cross-section and comparable 3D models are shown in Figure 12. Results are shown for a pellet with no defect and for the baseline $0.1 \mathrm{~mm}$ defect. The 3D model results are shown at the cladding interior at the pellet/pellet interface (pi) and waist (wi). Because the 2D model only represents the cross-section at a single location along the rod axis, results from that model are reported only at a single axial location. As shown in Figure 10, that location is denoted as wi, because it is somewhat equivalent to the location at the interior of the cladding adjacent to the defective pellet waist in the 3D model. To emphasize important effects with more clarity, Figure 12 shows a reduced set of quantities of interest, and shows those only for the cladding interior location.

From these plots, the following observations about the 3D vs. 2D cross-section representation of the defect can be made:

- Both with and without a defect, the results from the 2D model are reasonably close to the results from the 3D model at the cladding interior adjacent to the waist of the defective pellet (wi). As would be expected, they are not exactly the same, because the discrete pellet representation employed in the 3D model permits the capturing of pellet hourglassing effects, which is not possible in the 2D model. However, the 2D model captures the same trends in the response, and the differences in the $2 \mathrm{D}$ and $3 \mathrm{D}$ waist location results are quite small. It is also important to note that the shift in the response due to the defect relative to a pellet with no defect is very similar in the 2D model and in the $3 \mathrm{D}$ model at the waist location.

- Although the 2D model provides a very reasonable approximation of the response at the pellet waist in the 3D model, it only provides the response of the cross-section at one location along the axis of the rod. It has already been shown that the response varies significantly along the axis of the rod in the vicinity of the defect. For example, Figure 12 shows that the creep strains computed using the 3D model are significantly higher at the pellet/pellet interface than they are at the waist, and the 2D model cannot capture that effect.

Despite the limitations of the $2 \mathrm{D}$ model, it can be useful as an efficient screening tool because of its much lower computational cost. It provides a very good approximation of the response at the pellet waist for a constant-depth defect extending the entire length of the pellet. In addition, the fact the two models agree at the pellet waist helps provide confidence in the results from both models.

\section{Effect of Gaseous Swelling Model}

An important difference between the current work and previous efforts to model PCMI induced by missing pellet surface defects in BISON is that the currently employed model incorporates a physically based treatment of fuel gaseous swelling coupled with fission gas release. Figure 13 shows a comparison of time histories of quantities of interest produced with the physically based (Coupled) gaseous swelling model and results from an equivalent simulation using an empirical model for gaseous swelling as a function of temperature and burnup (MATPRO). A brief description of the two models and appropriate references was given in Section 3.4.

Throughout the irradiation history, the Coupled model results in consistently higher fuel swelling compared with the MATPRO model, as evident in the radial displacement history plots in Figure 13. This leads to higher cladding stresses and strains during mechanical contact between the fuel and cladding. In particular, during the time when the power is held high following the late-life high power ramp, cladding strains increase more significantly, as the Coupled model physically accounts for accelerated fuel swelling due to enhanced fission gas diffusion phenomena and associated gas bubble growth at higher temperatures [24, 25]. The significant role of gaseous swelling in fuel rod performance during fuel irradiation beyond the normal operating power and PCMI is well known $[22,23]$. In this respect, physically based models are preferred due to higher flexibility relative to the limited application range of empirical correlations [29, 30].

For the case shown here, the results obtained using the empirical MATPRO model have lower cladding stresses and strains during the same irradiation period compared to the Coupled model, and are thus less conservative. These results provide an initial insight into the importance of physically modeling gaseous swelling during off-normal situations and PCMI, although further investigation and comparison of code results with experimental data are advisable to better assess the accuracy of swelling models under such conditions. Initial validation of the Coupled model against irradiation experiments involving power ramps was reported in [24]. 

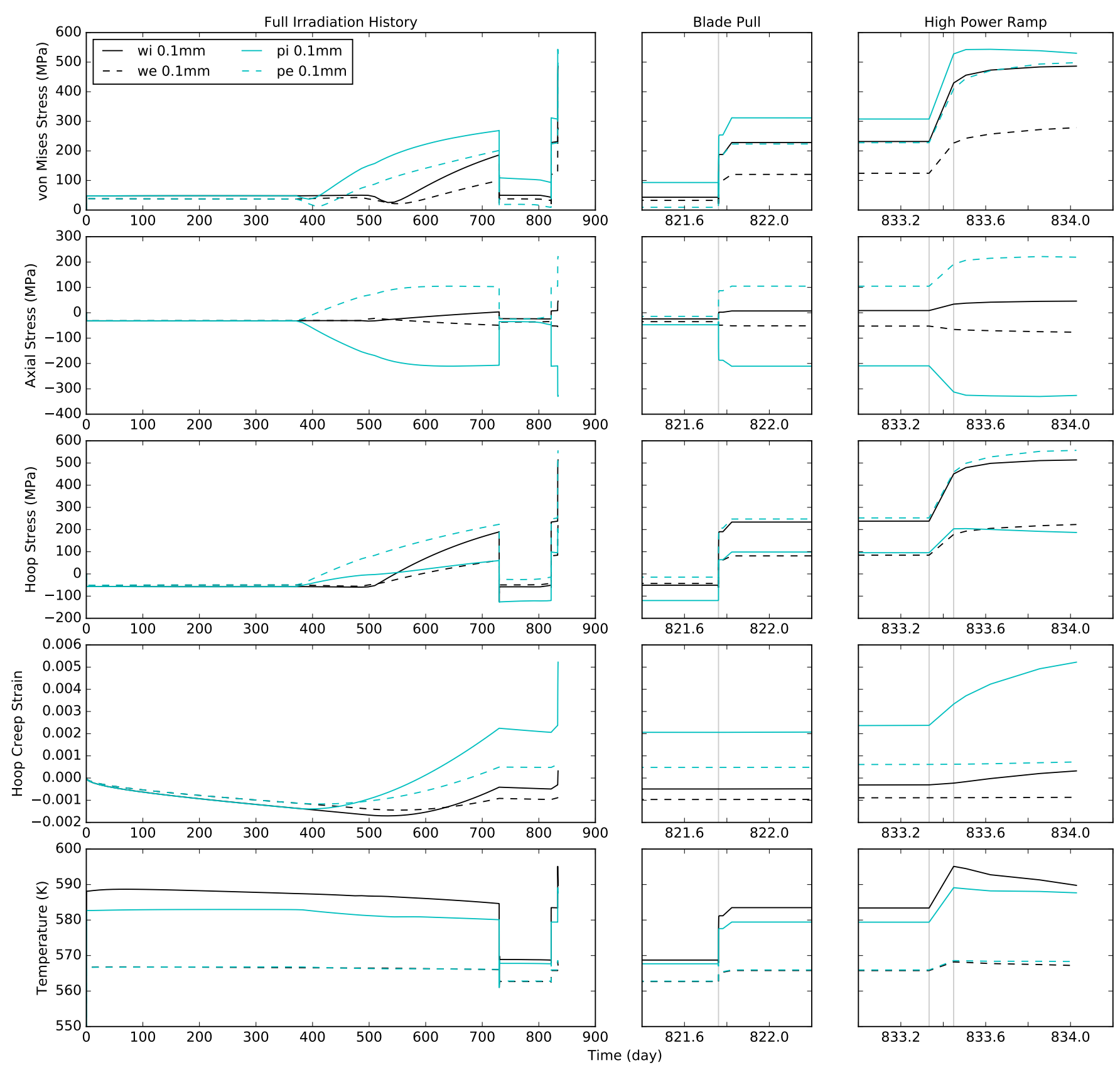

Figure 11: Time histories of von Mises stress, axial stress, hoop stress, hoop creep strain, and temperature at selected locations on the cladding interior and exterior for the baseline $0.1 \mathrm{~mm}$ defect pellet case. Quantities are reported at the the pellet/pellet interface at the cladding interior (pi) and exterior (pe) and at the defective pellet waist at the cladding interior (wi) and exterior (we), as shown in Figure 10. 

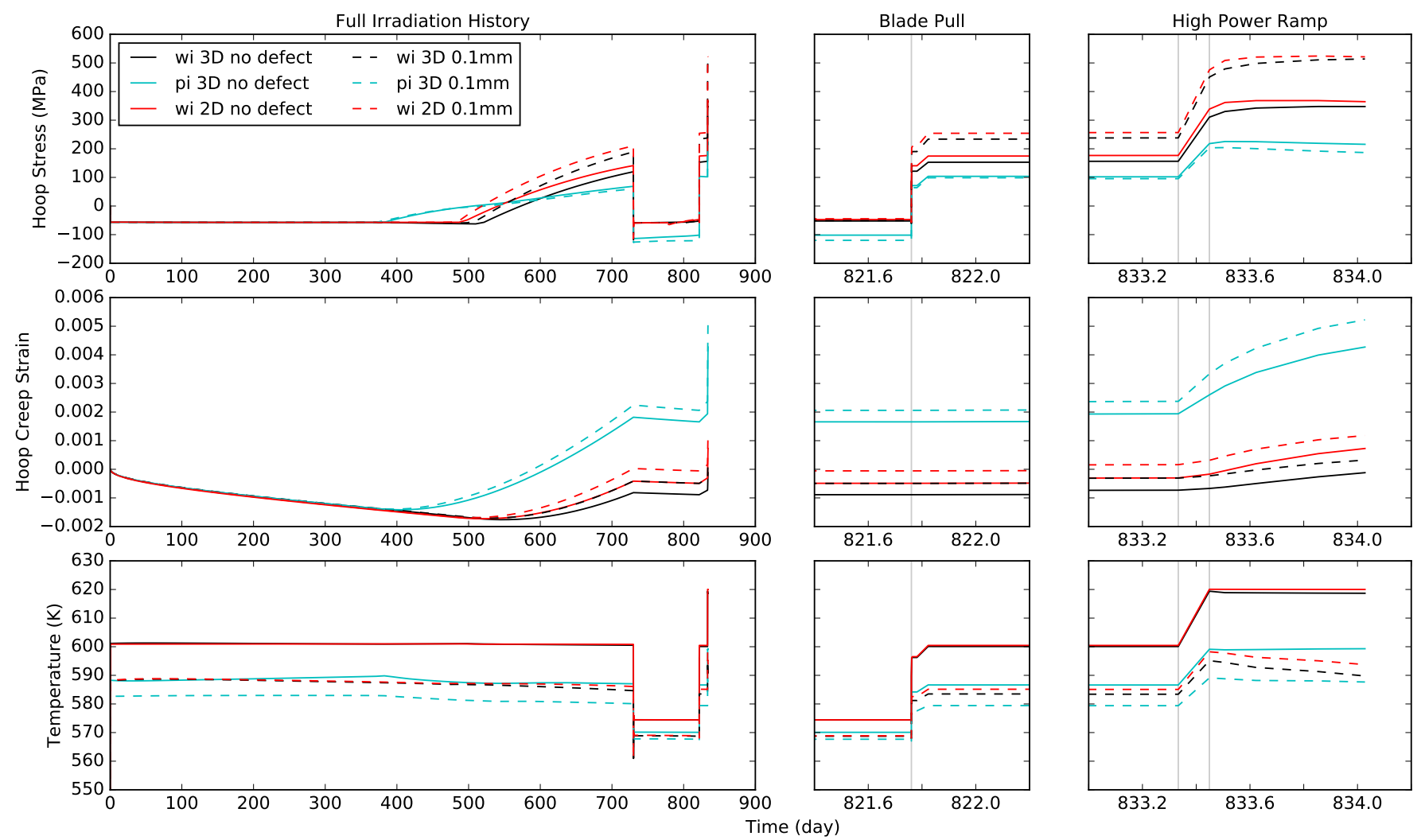

Figure 12: Time histories of hoop stress, hoop creep strain, and temperature at selected locations showing a comparison of the response with no defect and with a $0.1 \mathrm{~mm}$ defect using 3D and 2D models. Per Figure 10, results are at the cladding interior at the the pellet/pellet interface (pi) and at the defective pellet waist (wi).

\section{Effect of Defect Depth}

All results shown up to this point have been for a pellet with a $0.1 \mathrm{~mm}$ deep defect. To demonstrate the effect of the defect depth, three variants of the model were created with no defect, a $0.1 \mathrm{~mm}$ deep defect, and a $0.2 \mathrm{~mm}$ deep defect. The shape of the defect is identical for both defective pellet cases, consisting of a uniform-depth defect extending along entire length of the pellet as shown in Figure 2.

Figure 14 shows a comparison of the results for these three cases. These results are intuitive, in that the concentrations of stress and strain in the cladding in the vicinity of the defect increase in severity with increasing defect depth at both the pellet/pellet interface and at the defective pellet waist. This is true both for the blade pull transient and the ramp to high power.

\section{Effect of Defect Geometry}

The models of the defective pellet presented up to this point have represented the defect as a constant-depth slice extending along the entire axial length of the pellet caused by accidental machining. MPS defects can also be caused by chipping, which would result in a slanted defect that would only extend part-way down the axial length of the pellet. To investigate the effect of the defect geometry on the cladding response, additional 3D models have been developed with a defect represented by a cut with a slanted plane that intersects with the outer surface of the pellet half-way down the pellet.

Figure 15 shows the 3D finite element model used to represent the pellet with a slanted defect. Figure 15(a) shows the full mesh with the fuel and cladding. The geometry of the slanted defect does not permit use of symmetry conditions in the plane normal to the axial direction through the defective pellet, so this mesh contains the full defective pellet and two pellets above and below the defective pellet. Figure 15(b) shows a detailed view of the defective pellet without the clad. This mesh has the same density used in the other meshes, with approximately 280000 nodes and 31000 27-noded quadratic elements.

As for the other defect geometry, two defect depths were considered: $0.1 \mathrm{~mm}$ and $0.2 \mathrm{~mm}$, measured at the top of the pellet where the defect is the deepest. To illustrate the effect of the defect geometry, the cladding response is compared for the constant-depth and slanted defects in Figure 16. This shows contour plots of the von Mises stress in the cladding for these two cases, which both have $0.2 \mathrm{~mm}$ deep defects. As would be expected, the area of the zone affected by the defect is significantly smaller for the slanted defect, and its shape corresponds with the boundaries of the defect. The magnitude of the maximum stress on the inside of the cladding adjacent to the center of the slanted defect is almost as high as it is for the constant-depth defect. The slanted defect case does not show the narrow zone of high stresses at the border of the defect on the inside of the cladding that are seen in the straight defect case. This is likely 

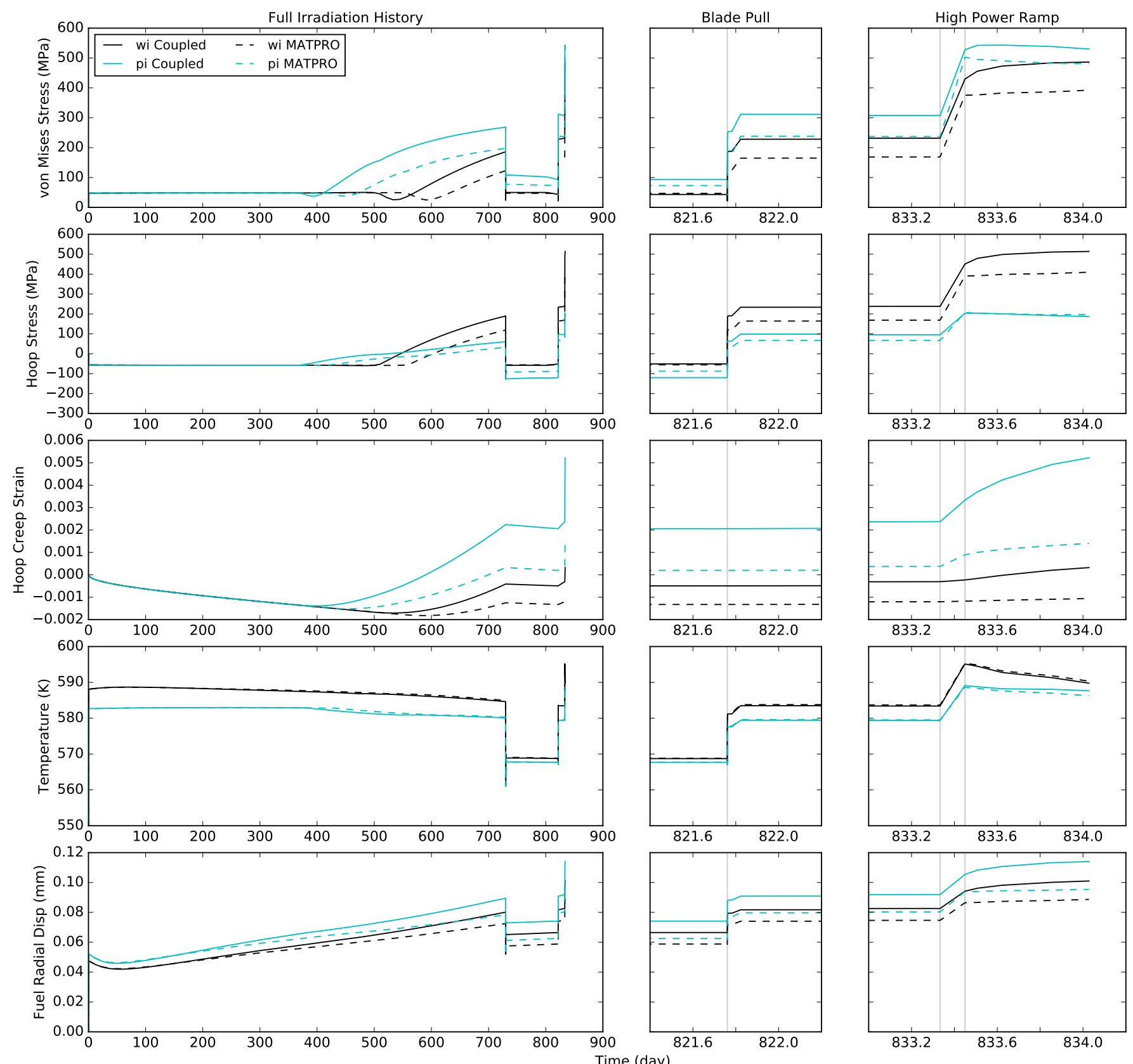

Figure 13: Time histories of von Mises stress, hoop stress, hoop creep strain, temperature, and fuel radial displacement at selected locations, showing a comparison of the response computed using the Coupled (gaseous swelling coupled with fission gas release) and MATPRO gaseous swelling model with a $0.1 \mathrm{~mm}$ defect. Quantities are reported at the the pellet/pellet interface at the cladding interior (pi) and at the defective pellet waist at the cladding interior (wi), as shown in Figure 10. Fuel radial expansion is reported at locations on the exterior of the fuel immediately adjacent to the pellet/pellet interface (pi) and waist (wi) locations in the cladding. 

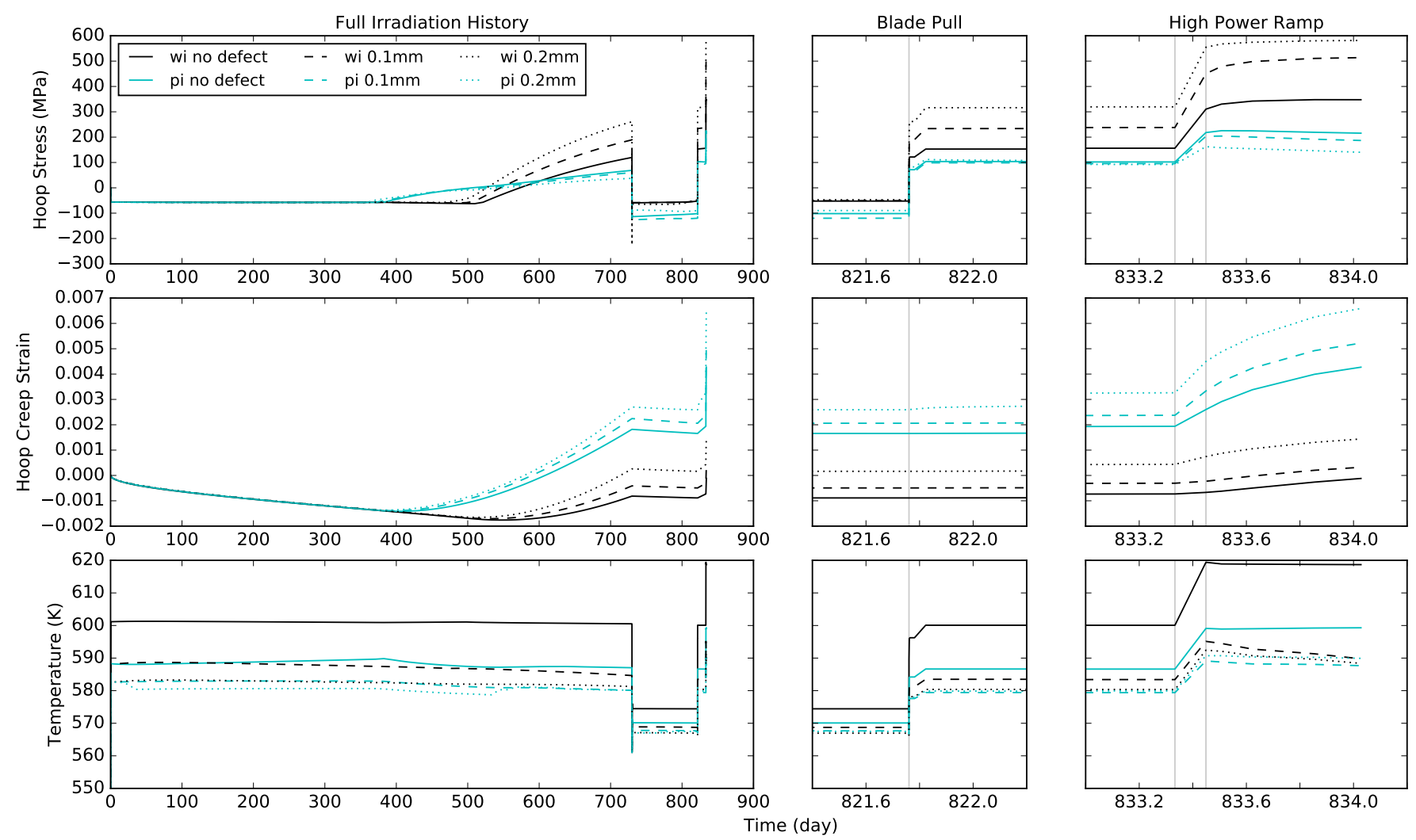

Figure 14: Time histories of hoop stress, hoop creep strain, and temperature at selected locations showing a comparison of the response with no defect, and with two depths of defect: $0.1 \mathrm{~mm}$ and $0.2 \mathrm{~mm}$. Per Figure 10, results are at the cladding interior at the the pellet/pellet interface (pi) and at the defective pellet waist (wi).

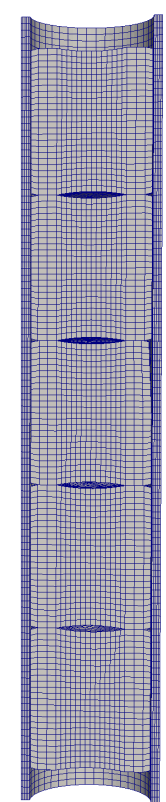

(a)

(b)

Figure 15: 3D finite element mesh of region of pellet with slanted defect. (a) View onto symmetry plane down axis of fuel rod showing fuel and cladding. Defect is on the upper right hand side of the middle pellet (b) View of defective pellet showing only the fuel mesh. due to differences in the way the mesh aligns with the edge of the defect.

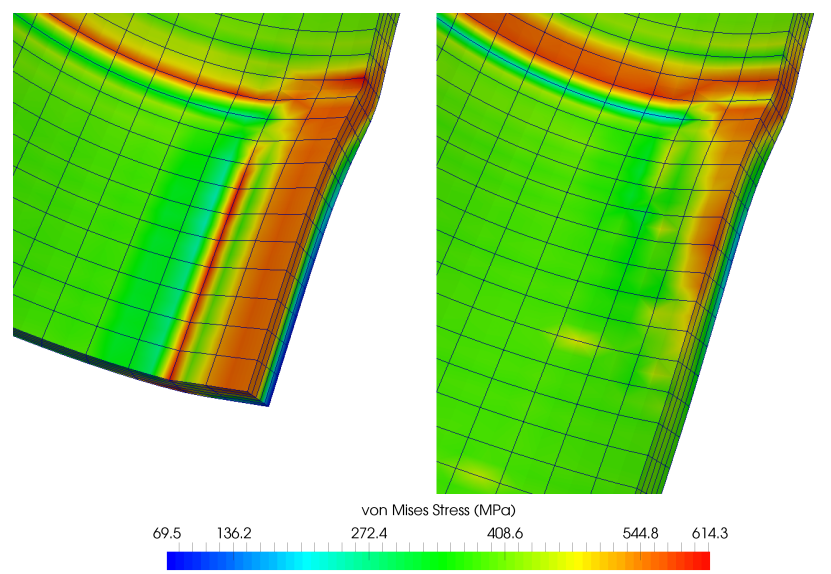

Figure 16: Comparison of cladding mechanical response for constant-depth (left) and slanted defect (right). Both defects have a maximum depth of $0.2 \mathrm{~mm}$. Contours of von Mises stress are shown from the same vantage point for both cases at the end of the analysis. Displacements are magnified 10x.

Figure 17 shows time histories of quantities of interest at selected locations in the cladding for the two depths of slanted defects along with the case with no defect (shown previously) for reference. These are shown at the pellet/pellet interface and at the pellet waist, as for other cases. Because the pellet waist is at the edge of the defect for this geometry, the stresses and 
strains are not significantly affected by the defect at that location. These quantities are also reported at a mid-defect location where stresses are maximal for this defect geometry. A notable finding of this analysis is that the mechanical response at that location is very similar to the response at the pellet waist for the constant-depth defect, which was shown in Figure 14. Defects that don't extend along the entire pellet have smaller zones of elevated stress, but the maximum magnitudes of those stresses are similar to those of a constant-depth defect.

It is important to note that the temperature response for the cases with the defective pellets differs from that of the constantdepth defect at the pellet/pellet interface. This is because there was more relative sliding between the fuel and cladding at this location because the fuel and cladding were fixed in the axial direction relative to each other at the bottom of the pellet stack rather than at the middle of the defective pellet. That location on the cladding had higher temperatures early in the analysis because it was adjacent to the pellet above the defective pellet, but as the fuel swelled, the fuel slid past that location, leaving it adjacent to a gap, resulting in lower temperatures. The transition is not smooth because of the way thermal contact is enforced at discrete points in the algorithm currently used. Increased mesh refinement or using a more sophisticated contact algorithm would decrease those effects.

\section{Conclusions}

An approach for modeling the coupled thermal/mechanical response of the fuel and cladding in the vicinity of a MPS defect has been described in detail and demonstrated on representative defective pellets in BWR rods under a variety of conditions. In this approach, a coupled physics fuel performance code is employed to model both the response of a full fuel rod using a 2D axisymmetric representation, and the response of the region of the defective pellet using either 2D cross-section or 3D models. Full time histories of quantities necessary to characterize the plenum gas behavior are transferred from the full rod model to the local models.

Using a multidimensional fuel performance code to compute both the full rod response and the local response enables the full set of physics and behavior models typically used in fuel performance simulations to be used in studying the effects of local geometric features such as MPS defects. Because the BISON code can be run on parallel computers, detailed 3D modeling of those local features is quite feasible.

Efforts were made to include a complete set of physics and behavior models, and the current work represents a significant improvement over previous efforts to model this problem in BISON. There are still some important effects that were not included because these features are still in development in BISON for this application. Notably, fuel fracture and creep were not modeled in either the full rod or the local region model. In addition, friction was not included in the mechanical contact interactions. While most current BWR cladding designs incorporate a thin zirconium liner, the current model does not include the effects of that liner. Finally the radial variation in the power is not included in the local models because of the complexities involved in accounting for the defective pellet geometry. Ongoing work is underway to include these effects in modeling MPS defects.

The approach described here has been demonstrated on postulated MPS defects in a representative BWR rod subjected to an irradiation history that consists of a base irradiation in a position away from control blades, a fuel shuffle to a position adjacent to a control blade, a blade pull event, and a ramp to high power. The response of the cladding in positions adjacent to the defect was studied in detail under those conditions.

The response of the baseline 3D model was compared with an equivalent $2 \mathrm{D}$ generalized plane strain model. While there was reasonable agreement between the 3D and 2D local models, the 2D local model can only reasonably represent the response at the waist of the defective pellet, and misses important effects that can only be captured with a full 3D local model. To demonstrate the importance of using the coupled gaseous swelling model used in the present work, it was compared with an empirical model used in previous work, which was shown to produce less conservative results for the conditions studied here. Parametric studies were performed to show the effect of the defect depth and geometry on the cladding response. Deeper defects resulted in stress and strain concentrations of increasing severity. Defects that do not extend along the entire pellet length have smaller regions of elevated stress, but the maximum stress is similar to that of a constant-depth defect. The modeling approach employed here is very flexible, and can represent arbitrary defect geometries.

The current work has focused on accurately computing the thermal and mechanical response of the cladding in the vicinity of the defect, but has not attempted to assess whether failure occurs. The thermal and mechanical quantities of interest computed and presented here are very helpful in understanding the cladding response, and must be computed to make a failure prediction. However, these are not by themselves sufficient to predict failure due to stress-corrosion cracking, as this is also dependent on the chemical environment. The modeling tools demonstrated here are currently being extended to permit their use for failure prediction.

\section{Acknowledgments}

This work was funded by the US Department of Energy under the Nuclear Energy Advanced Modeling and Simulation (NEAMS) and Consortium for the Advanced Simulation of Light Water Reactors (CASL) programs. The submitted manuscript has been authored by a contractor of the U.S. Government under Contract DE-AC07-05ID14517. Accordingly, the U.S. Government retains a non-exclusive, royalty free license to publish or reproduce the published form of this contribution, or allow others to do so, for U.S. Government purposes.

\section{References}

[1] F. Groeschel, G. Bart, R. Montgomery, and S. K. Yagnik. Failure root cause of a PCI suspect liner fuel rod. In IAEA Techincal Meeting on Fuel Failure in Water Reactors: Causes and Mitigation, Bratislave, Slovakia, Jun 17-21 2002 

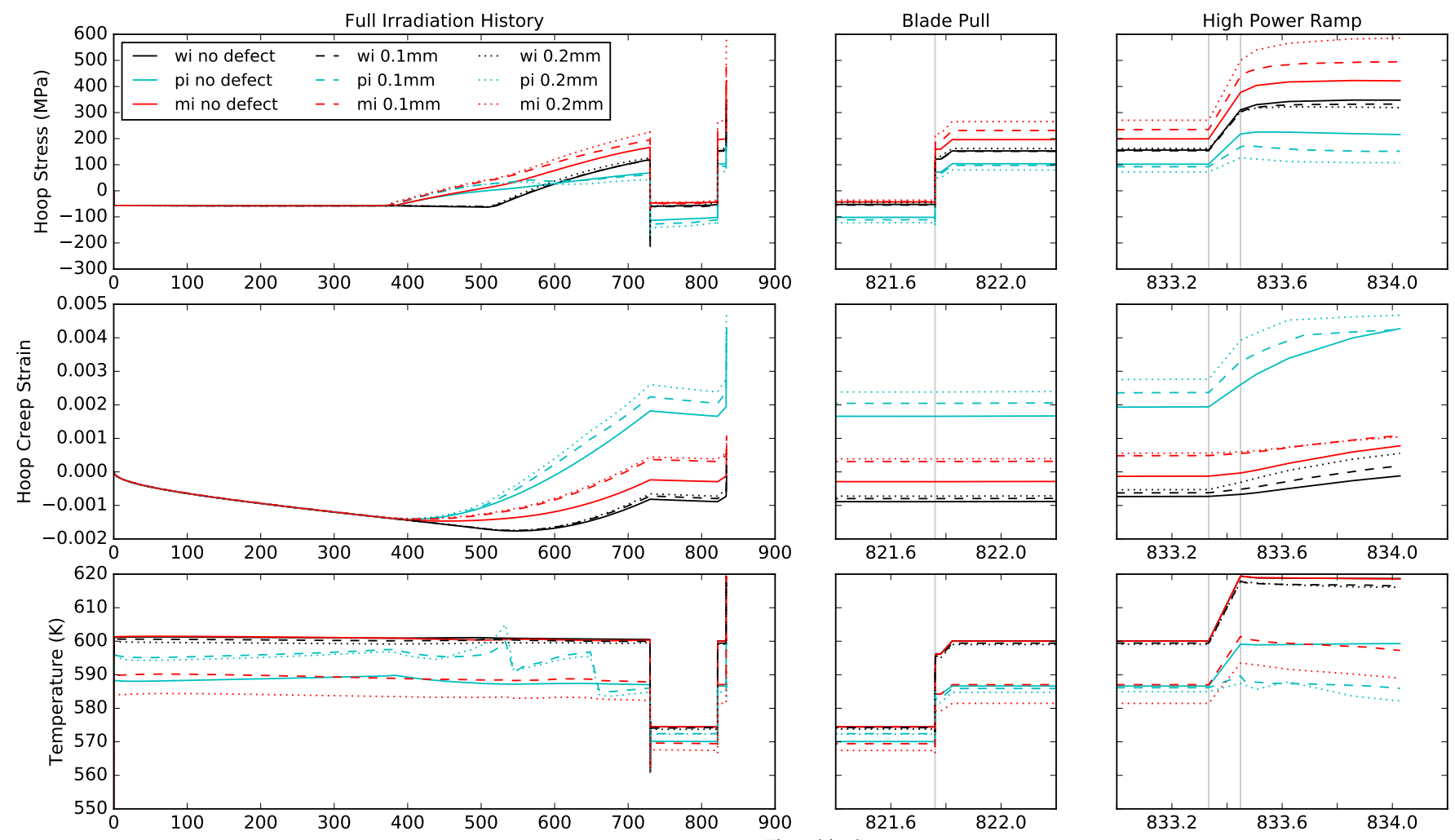

Figure 17: Time histories of hoop stress, hoop creep strain, and temperature at selected locations showing a comparison of the response with no defect, and with two depths of slanted defect: $0.1 \mathrm{~mm}$ and $0.2 \mathrm{~mm}$. Per Figure 10, results are at the cladding interior at the the pellet/pellet interface (pi) and at the defective pellet waist (wi), as well as at an additional location approximately $1.4 \mathrm{~mm}$ down from the top of the defective pellet where the stress is maximal on the cladding interior (mi). 
[2] A. Garlick and P. D. Wolfenden. Fracture of zirconium alloys in iodine vapour. Journal of Nuclear Materials, 41:274-292, 1971.

[3] B. Cox. Pellet-clad interaction (PCI) failures of zircomium alloy fuel cladding - A review. Journal of Nuclear Materials, 172:249-292, 1990.

[4] G. A. Potts. Recent GE BWR fuel experience. In Proceedings of the 1997 International Topical Meeting on LWR Fuel Performance, pages 261-271, Portland, Oregon, Mar 2-6 1997.

[5] D. Dangouleme, V. Inozemtsev, K. Kamimura, J. Killeen, A. Kucuk, V. Novikov, V. Onufriev, and M. Tayal. IAEA review on fuel failures in water cooled reactors. In Proceedings of 2010 LWR fuel performance, Orlando, FL, USA, Sep 26-29 2010.

[6] M. Billaux and H. Moon. Pellet-cladding mechanical interaction in boiling water reactors. In Proceedings of Pellet-Clad Interaction in Water Reactor Fuels, pages 43-52, Aix-en-Provence, France, Mar 9-11 2004.

[7] Y. Aleshin, C. Beard, G. Mangham, D. Mitchell, E. Malek, and M. Young. The effect of pellet and local power variations on PCI margin. In Proceedings of Top Fuel, 2010, Orlando, FL, USA, Sep 26-29 2010.

[8] S. Nesbit, M. Kennard, and S. Yagnik. Use of core analyses in assessments of fuel failure risk due to pellet-cladding interaction. In Proceedings of Advances in Nuclear Fuel Management IV, Hilton Head Island, South Carolina, Apr 12-15 2009.

[9] G. A. Berna, C. E. Beyer, K. L. Davis, and D. D. Lanning. FRAPCON3: A Computer Code for the Calculation of Steady-state, ThermalMechanical Behavior of Oxide Fuel Rods for High Burnup. Technical Report NUREG/CR-6534 Vol. 2, PNNL-11513, 1997.

10] K. Lassmann, A. Schubert, and J. van de Laar. TRANSURANUS handbook. Technical Report Document Number Version 1 Modification 1 Year 2003 (V1M1J2003), September 2003.

[11] W. J. Kilgour, J. A. Turnbull, R. J. White, A. J. Bull, P. A. Jackson, and I. D. Palmer. Capabilities and validation of the ENIGMA fuel performance code. In Proceedings of the ENS Meeting on LWR Fuel Performance, Avignon, France, 1991.

[12] Y. Rashid, R. Dunham, and R. Montgomery. Fuel analysis and licensing code: FALCON MOD01. Technical Report EPRI 1011308, Electric Power Research Institute, December 2004.

[13] W. Lyon, R. Montgomery, J. Rashid, and S. Yagnik. PCI analysis of fuel rod failure prediction using FALCON. In Proceedings of Top Fuel 2009, Paris, France, Sep 6-10 2009.

[14] G. Khvostov, W. Lyon, and M.A. Zimmermann. Application of the FALCON code to PCI induced cladding failure and the effects of missing pellet surface. Annals of Nuclear Energy, 62:398-412, 2013.

[15] J. S. Lee, J. S. Yoo, H. K. Kim, D. Mitchell, and Y. Aleshin. The mechanical behavior of pellet-cladding with the missing chip under PCMI loadings during power ramp. In 2007 International LWR Fuel Performance Meeting - TopFuel, San Francisco, CA, September 30-October 32007.

[16] R. L. Williamson, J. D. Hales, S. R. Novascone, M. R. Tonks, D. R. Gaston, C. J. Permann, D. Andrs, and R. C. Martineau. Multidimensional multiphysics simulation of nuclear fuel behavior. J. Nucl. Mater., 423:149163, 2012.

[17] B. W. Spencer, J. D. Hales, S. R. Novascone, and R. L. Williamson. 3D Simulation of Missing Pellet Surface Defects in Light Water Reactor Fuel Rods. In Transactions of Top Fuel Reactor Fuel Performance 2012, Manchester, United Kingdom, September 2-6, 2012.

[18] J. D. Hales, R. L. Williamson, S. R. Novascone, D. M. Perez, B. W. Spencer, and G. Pastore. Multidimensional multiphysics simulation of TRISO particle fuel. J. Nucl. Mater., 443:531-543, 2013.

[19] D. Gaston, C. Newman, G. Hansen, and D. Lebrun-Grandié. MOOSE: A parallel computational framework for coupled systems of nonlinear equations. Nucl. Eng. Design, 239:1768-1778, 2009.

[20] K. Lassmann, C. O'Carroll, J. van de Laar, and C. T. Walker. The radia distribution of plutonium in high burnup $\mathrm{UO}_{2}$ fuels. J. Nucl. Materials, 208:223-231, 1994

[21] C. M. Allison, G. A. Berna, R. Chambers, E. W. Coryell, K. L. Davis, D. L. Hagrman, D. T. Hagrman, N. L. Hampton, J. K. Hohorst, R. E. Mason, M. L. McComas, K. A. McNeil, R. L. Miller, C. S. Olsen, G. A. Reymann, and L. J. Siefken. SCDAP/RELAP5/MOD3.1 code manual, volume IV: MATPRO-A library of materials properties for light-water-reactor accident analysis. Technical Report NUREG/CR-6150, EGG-2720, Idaho National Engineering Laboratory, 1993.

[22] IAEA. Improvement of Computer Codes Used for Fuel Behaviour Simulation (FUMEX-III): Report of a Coordinated Research Project 2008-
2012. Technical Report IAEA-TECDOC-1697, International Atomic Energy Agency, 2013.

[23] P. Van Uffelen, M. Sheindlin, V. Rondinella, and C. Ronchi. On the relations between the fission gas behaviour and the pellet-cladding mechanical interaction in LWR fuel rods. In Proc. of the International Seminar on Pellet-Clad Interaction in Water Reactor Fuels, Aix-en-Provence, France, March 9-11, 2004.

[24] G. Pastore, L. Luzzi, V. Di Marcello, and P. Van Uffelen. Physics-based modelling of fission gas swelling and release in $\mathrm{UO}_{2}$ applied to integral fuel rod analysis. Nucl. Engrg. Design, 256:75-86, 2013.

[25] G. Pastore, L. P. Swiler, J. D. Hales, S.R. Novascone, D. M. Perez, B. W. Spencer, L. Luzzi, P. Van Uffelen, and R. L. Williamson. Uncertainty and sensitivity analysis of fission gas behavior in engineering-scale fuel modeling. Journal of Nuclear Materials, 456:398-408, 2015.

[26] M. Limbäck and T. Andersson. A model for analysis of the effect of final annealing on the in- and out-of-reactor creep behavior of zircaloy cladding. In Zirconium in the Nuclear Industry: Eleventh International Symposium, ASTM STP 1295, pages 448-468, 1996.

[27] Y. Matsuo. Thermal creep of zircaloy-4 cladding under internal pressure. Journal of Nuclear Science and Technology, 24(2):111-119, February 1987.

[28] N. E. Hoppe. Engineering model for zircaloy creep and growth. In Proceedings of the ANS-ENS International Topical Meeting on LWR Fuel Performance, pages 157-172, Avignon, France, April 21-24, 1991.

[29] C. Calvin and D. Nowak. High performance computing in nuclear engineering. In D. G. Cacuci, editor, Handbook of Nuclear Engineering, volume 12, pages 1449-1517. Springer Science + Business Media, LLC., New York, NY, USA, 2010.

[30] A. Karahan and M. S. Kazimi. On the significance of modeling nuclear fuel behavior with the right representation of the physical phenomena. Nuclear Engineering and Design, 241:484-491, 2011. 